\begin{tabular}{|c|c|c|}
\hline & Int.J.Curr.Microbiol.App.Sci (2021) 10(10): 108-117 & \\
\hline EXCELLENT & $\begin{array}{l}\text { International Journal of Current Microbiology and Applied Sciences } \\
\text { ISSN: 2319-7706 Volume } 10 \text { Number } \mathbf{1 0}(\mathbf{2 0 2 1 )} \\
\text { Journal homepage: http://www.ijcmas.com }\end{array}$ & $\begin{array}{l}9 \\
39\end{array}$ \\
\hline PUBLISHERS & & www.ijemas.com \\
\hline
\end{tabular}

\title{
Seasonal Abundance of Brown Plant Hopper, Nilaparvata lugens (Stal) in Rice and Correlation of Abiotic Factors Under Pune Region
}

\author{
Amruta Deshmukh*, S. B. Kharbade, N. D. Tamboli, V. A. Sthool and S. V. Bagade
}

Department of Agriculture Meteorology, College of Agriculture, Pune-411005, Maharashtra, India

*Corresponding author

\section{A B S T R A C T}

Keywords

Brown plant hopper, Rice, weather parameters, transplanting dates, Correlation

Article Info

\section{Accepted:}

05 September 2021

Available Online:

10 October 2021
An agro-meteorological investigation was undertaken on "Crop weather pest relationship and validation of DSSAT model for rice varieties under different transplanting dates" during kharif, 2016 and 2017 at Agricultural Research Station Farm, Vadgaon Maval, Dist. Pune, under Mahatma Phule Krishi Vidyapeeth, Rahuri, University. An experiment was laid out in split plot design with three replications. The treatment comprised of four sowing dates viz., $\mathrm{S}_{1}: 26^{\text {th }} \mathrm{MW}$ (25 June-1 July), $\mathrm{S}_{2}: 28^{\text {th }}$ MW (9 July-15 July), $S_{3}: 30$ MW (23 July-29 July) and $S_{4}: 32^{\text {nd }}$ MW (06 August -12 August) as main plot treatments and four varieties viz., $\mathrm{V}_{1}$ : VDN-99-29 (Phule Samruddhi), $\mathrm{V}_{2}$ : VDN-3-51-18 (Indrayani), $\mathrm{V}_{3}$ : IET-13549 (Bhogavati) and $\mathrm{V}_{4}$ : RDN-99-1 (Phule Radha) as sub plot treatments. Average number of brown plant hopper showed highly significant positive correlation with maximum temperature $\left(0.902^{* *}, 0.627 * *\right)$, positive correlation with morning humidity $\left(0.534,0.789^{*}\right)$, evening humidity $\left(0.678^{*}, 0.551\right)$ and bright sunshine hours $(0.345,0.483)$. While minimum temperature $(-0.567,-0.543)$ and rainfall $(-0.302,-0.307)$ showed negative correlation with brown plant hopper on $26^{\text {th }} \mathrm{MW}, 28^{\text {th }} \mathrm{MW}, 30^{\text {th }} \mathrm{MW}$ and $32^{\text {nd }} \mathrm{MW}$ transplanting dates. During both the years of 2016 and 2017, respectively.

\section{Introduction}

Rice (Oryza sativa L.) is a staple food for more than 65 per cent of the people and it provides employment and livelihood security to 70 per cent of Indian population. India grows rice in highly diverse conditions starting from below sea levels to hill as high as $>2000$ meters. India ranks first in area with about $44 \mathrm{~m}$. ha under rice and second in production with 104.8 million tonnes with an average productivity of $2390 \mathrm{~kg} \mathrm{ha}^{-1}$ Different transplanting times affects crop growth and yield considerably. Therefore, optimum transplanting times for better crop yield is essential. The Brown plant hopper is the monophagous, small or tiny insect with 2.0 to $3.5 \mathrm{~mm}$ body length. It is a brownish colour insect which feed on the phloem of plant. Adult and nymphs, both damage to the phloem 
of rice plant. Though this insect is known to occur in Asia since late forties, it was earlier only a minor pest of rice. Serious outbreaks of BPH were reported in various parts of India during early $70 \mathrm{~s}$. BPH damages plant directly by sucking to plant sap and indirectly by transmitting viral disease like grassy stunt and ragged stunt. BPH caused economic damage by sucking phloem sap which in turn leads to "hopper burn" and sever yield loss (Rao et al., 2003). Both the nymphs and adults of these hoppers suck the sap from phloem and xylem resulting in drying up of the rice plant. At early infestation, round, yellow patches appear, which soon turn brownish due to the drying up of the plants (Ling, 1975). Agriculture consumes 52 per cent of the total insecticides in India, and intern rice crop alone accounts for 17 per cent of it. About 50 per cent of Indian rice farmers use insecticides ranging from one to six applications per crop season against Stem borers, Brown plant hopper, White backed plant hopper and Leaf folder. The BPH, Nilaparvata lugens (Stal.) is one of most economic pests of rice causes significant losses in yield of the crop.

Therefore, to overcome the insect pest damage by forewarning the pest incidence and to obtain higher yields, it is necessary to find out the optimum transplanting time and validate the crop models. Keeping this in view, the field experiment was planned in Kharif 2016 and 2017.

\section{Materials and Methods}

The present experiments related investigation entitled, "Crop weather pest relationship and validation of DSSAT model for Rice varieties under different transplanting dates" were conducted at Agriculture Research Station Farm, Vadgaon Maval, Tal. Dist. (M.S.) during kharif seasons of 2016-17 and 2017-18, to identify optimum transplanting date for Rice, to develop crop weather relationships, to develop pest weather relations and to validate the DSSAT model. An experiment was laid out in split plot design with three replications. The treatment comprised of four sowing dates viz., $\mathrm{S}_{1:} 26^{\text {th }} \mathrm{MW}$ (25 June-1 July), $\mathrm{S}_{2:} 28^{\text {th }}$ MW (9 July-15 July), S: 30 MW (23 July-29 July) and $\mathrm{S}_{4}$ : $32^{\text {nd }}$ MW (06 August -12 August) as main plot treatments and four varieties viz., $\quad \mathrm{V}_{1}$ : VDN-99-29 (Phule Samruddhi), $\mathrm{V}_{2:}$ VDN-3-51-18 (Indrayani), $\mathrm{V}_{3}$ : IET-13549 (Bhogavati) and $\mathrm{V}_{4}$ : RDN-99-1 (Phule Radha) as sub plot treatments.

\section{Experimental Details}

The experiment was conducted in a split -plot design with three replications and sixteen treatment combinations were formed considering different varieties and transplanting. The details are listed below.

Name of crop : Rice

Varieties : 1. VDN-99-29 (Phule Samrudhi)

VDN-3-51-18 (Indrayani)

IET-13549 (Bhogavati)

RDN-99-1 (Phule Radha)

Number of treatments : 16 combinations

Number of replications : 3

Number of plots : 48

Experimental Design : Split plot Design

\section{Plot size}

a. Gross Plot: $4.80 \mathrm{~m}$ x $3.60 \mathrm{~m}$

b. Net Plot:: $4.0 \mathrm{~m}$ x $3.0 \mathrm{~m}$

Spacing : $20 \mathrm{~cm} \mathrm{x} 15 \mathrm{~cm}$ 
Seed rate $: 20 \mathrm{~kg} \mathrm{ha}^{-1}$

Fertilizer dose : 175: 60: 60 NPK kg ha ${ }^{-1}$

\section{Seasons}

Kharif, 2016

Kharif, 2017

Place of research work : Agricultural Research Station Farm, Vadgaon Maval, Tal. Maval, Dist. Pune

\section{Transplanting dates}

$\mathrm{S}_{1}: 26^{\text {th }}$ MW (25 June-1 July)

$\mathrm{S}_{2}: 28^{\text {th }}$ MW (16 July-15July)

$\mathrm{S}_{3}: 30 \mathrm{MW}$ (23 July-29July)

$\mathrm{S}_{4}: 32 \mathrm{MW}$ (13Auguest -19Auguest)

\section{Results and Discussion}

\section{Brown plant hopper}

Brown plant hopper infests the rice crop at all the stages of crop growth. As a result of feeding by both nymphs and adults at the base of the tillers, plants turn yellow and dry up rapidly. At early infestation, round, yellow patches appear, which soon turn brownish due to the drying up of the plants. This condition is called 'hopper burn'. Temperature is a critical factor in the life activities of the insect. The hatchability and survival rate are the highest around $25^{\circ} \mathrm{C}$. Eggs are very sensitive to desiccation and soon shrivel when the host plant starts wilting. The population growth of brown plant hopper is maximum at a temperature range of $28^{\circ}$ to $30^{\circ} \mathrm{C}$.

The incidence of brown plant hopper was recorded on all rice varieties during the year
2016, across all transplanting times on rice. Normally, the temperature also showed increasing trend from September onwards and has the lowest during November. Hence, with increasing temperature certainly resulted in increase in the infestation of brown plant hopper. Morning and evening relative humidity was also higher in month of September which resulted in increase in brown plant hopper population. When minimum temperature and rainfall increased, there was corresponding decrease in rice brown plant hopper Subhash et al., (2016) reported that brown plant hopper appeared on rice crop during the second week of September. During the transplanting times of $26^{\text {th }} \mathrm{MW}$ the brown plant hopper populations ranged between 4.4 and 39.93 on rice varieties VDN-99-29 $\left(\mathrm{V}_{1}\right)$, VDN-3-51-18 $\left(\mathrm{V}_{2}\right)$, IET13549 $\left(\mathrm{V}_{3}\right)$ and RDN99-1 $\left(\mathrm{V}_{4}\right)$. The highest populations of brown plant hopper 39.93 were recorded on RDN-991. During the transplanting times of $28^{\text {th }} \mathrm{MW}$, the brown plant hopper populations varied from 3.9 to 29.07 on rice varieties VDN-99-29 $\left(\mathrm{V}_{1}\right)$, VDN-3-51-18 $\left(\mathrm{V}_{2}\right)$, IET-13549 $\left(\mathrm{V}_{3}\right)$ and RDN-99-1 $\quad\left(\mathrm{V}_{4}\right)$. (Table.2)The highest populations of brown plant hopper 29.07 were recorded on RDN-99-1.During the transplanting times of $30 \mathrm{MW}$, the brown plant hopper populations varied from 3.8 to 40.30 on rice varieties viz., VDN-99-29 $\left(\mathrm{V}_{1}\right)$, VDN-3-51-18 $\left(\mathrm{V}_{2}\right)$, IET13549 $\left(\mathrm{V}_{3}\right)$ and RDN99-1 $\left(\mathrm{V}_{4}\right)$. The highest population of brown plant hopper 40.30 was recorded on RDN-991 . During the transplanting times of $32^{\text {nd }} \mathrm{MW}$, the brown plant hopper populations varied from 3.1 to 56.50 on rice varietiesVDN-99-29 $\left(\mathrm{V}_{1}\right)$, VDN-3-51-18 $\left(\mathrm{V}_{2}\right)$, IET13549 $\left(\mathrm{V}_{3}\right)$ and RDN-99-1 $\left(\mathrm{V}_{4}\right)$. The highest population of brown plant hopper 56.50 was recorded on RDN-99-1.

The incidence of brown plant hopper was recorded on all rice varieties during the year 2017, across all transplanting times on rice. During the transplanting times of $26^{\text {th }} \mathrm{MW}$, 
the brown plant hopper populations ranged between 4.1 and 46.30 on rice VDN-99-29 $\left(\mathrm{V}_{1}\right)$, VDN-3-51-18 $\left(\mathrm{V}_{2}\right)$, IET13549 $\left(\mathrm{V}_{3}\right)$ and RDN-99-1 $\left(\mathrm{V}_{4}\right)$. The highest populations of brown plant hopper 46.30 were recorded on RDN-99-1. During $32^{\text {nd }}$ MW. During the transplanting times of $28^{\text {th }} \mathrm{MW}$, the brown plant hopper populations varied from 3.2 to 24.18 on rice varieties viz, VDN-99-29 $\left(\mathrm{V}_{1}\right)$, VDN-3-51-18 ( $\left.\mathrm{V}_{2}\right)$, IET13549 $\left(\mathrm{V}_{3}\right)$ and RDN99-1 $\left(\mathrm{V}_{4}\right)$.(Table.3) The highest population of brown plant hopper 24.18 was recorded on RDN-99-1 during 32 ${ }^{\text {nd }}$ MW. During the transplanting times of $30 \mathrm{MW}$, the brown plant hopper populations varied from 4.4 to 41.2 on rice VDN-99-29 $\left(\mathrm{V}_{1}\right)$, VDN-3-51-18 $\left(\mathrm{V}_{2}\right)$, IET13549 $\left(\mathrm{V}_{3}\right)$ and RDN-99-1 $\left(\mathrm{V}_{4}\right)$ varieties.

Among the rice varieties, for the year of 2016 and 2017 higher incidence was recorded RDN-99-1 and minimum was recorded on VDN-99-29 i.e Phule samruddhi. Similar results were reported by Rai and Khan (2002), Wains et al., (2010), Chaudhary and
Raghuraman (2014) and Subhash et al., (2016).

Influence of weather parameters on rice brown plant hopper

The correlation of meteorological parameters with incidence of rice brown plant hopper was studied in 2016-17. The correlation coefficient (r) of brown plant hopper on rice with the weather parameters are presented in (Table.2) Average number of brown plant hopper showed significant positive correlation with maximum temperature $\left(\mathrm{r}=0.803^{*}, 0.902 * *\right.$, $0.789 *, 0.899 *)$, morning and evening relative humidity's and bright sunshine hrs $(\mathrm{r}=0.234$, $0.345,0.457,0.567)$ during the year of 2016 , Whereas brown plant hopper population showed negative correlation with rainfall and minimum temperature during $26 \mathrm{MW}, 28$ MW, $30 \mathrm{MW}$ and $32 \mathrm{MW}$ transplanting times $(\mathrm{r}=-0.142,-0.302,-0.450$ and -0.845$),(\mathrm{r}=-$ $0.789,-0.567,-0.812 *$ and -0.567$)$, during the year of 2016 .

Table.1 Correlation of weather parameters and rice brown plant hopper (2016-17)

\begin{tabular}{|l|c|c|c|c|c|c|}
\hline \multirow{2}{*}{ Sowing times } & \multicolumn{7}{|c|}{ Correlation coefficient values } \\
\cline { 2 - 6 } & \multicolumn{7}{|c|}{$\mathbf{2 0 1 6}$} \\
\cline { 2 - 6 } & $\mathbf{R F}$ & Tmax & Tmin & R H I & R H II & BSS \\
\hline $\mathrm{S}_{1}-26^{\text {th }} \mathrm{MW}$ & -0.142 & $0.803^{*}$ & $-0.789^{*}$ & 0.567 & $0.788^{*}$ & 0.234 \\
\hline $\mathrm{S}_{2}-28^{\text {th }} \mathrm{MW}$ & -0.302 & $0.902^{*}$ & -0.567 & 0.534 & $0.678^{*}$ & 0.345 \\
\hline $\mathrm{S}_{3}-30^{\text {th }} \mathrm{MW}$ & -0.450 & $0.789^{*}$ & $-0.812^{*}$ & 0.456 & 0.234 & 0.456 \\
\hline $\mathrm{S}_{4}-32^{\text {th }} \mathrm{MW}$ & $-0.845^{*}$ & $0.899^{*}$ & -0.567 & 0.345 & 0.456 & 0.567 \\
\hline & & \multicolumn{2}{|c|}{$\mathbf{2 0 1 7}$} & & \\
\hline $\mathrm{S}_{1}-26^{\text {th }} \mathrm{MW}$ & -0.192 & $0.874^{*}$ & $-0.637^{*}$ & 0.568 & $0.615^{*}$ & 0.224 \\
\hline $\mathrm{S}_{2}-28^{\text {th }} \mathrm{MW}$ & 0.307 & $0.627^{*}$ & -0.543 & $0.789^{*}$ & 0.551 & 0.183 \\
\hline $\mathrm{S}_{3}-30^{\text {th }} \mathrm{MW}$ & $-0.668^{*}$ & $0.724^{*}$ & $-0.745^{*}$ & $0.943^{*}$ & $0.811^{*}$ & 0.400 \\
\hline $\mathrm{S}_{4}-32^{\text {th }} \mathrm{MW}$ & $-0.746^{*}$ & $0.678^{*}$ & -0.593 & 0.567 & 0.305 & 0.482 \\
\hline
\end{tabular}


Table.2 Seasonal incidence of brown plant hopper association with rice (2016)

\begin{tabular}{|c|c|c|c|c|c|c|c|c|c|c|c|c|c|c|c|c|}
\hline \multirow{7}{*}{$\begin{array}{c}\mathrm{S}_{1}-26^{\text {th }} \\
M W\end{array}$} & Sr. & Variety & \multicolumn{11}{|c|}{ Mean of observations taken on SMW } & \multirow{2}{*}{\multicolumn{2}{|c|}{ Range }} & \multirow[t]{2}{*}{ Mean } \\
\hline & No & & 28 & 29 & 30 & 31 & 32 & 33 & 34 & 35 & 36 & 37 & 38 & & & \\
\hline & \multicolumn{13}{|c|}{ No. of insects /40 hills } & Min & Max & \\
\hline & 1 & $\begin{array}{c}\text { Phule } \\
\text { Samrudhi }\end{array}$ & $\begin{array}{c}4.4 \\
(2.32)\end{array}$ & $\begin{array}{c}5.80 \\
(2.61)\end{array}$ & $\begin{array}{l}10.28 \\
(3.36)\end{array}$ & $\begin{array}{c}13.93 \\
(3.8)\end{array}$ & $\begin{array}{l}20.90 \\
(4.68)\end{array}$ & $\begin{array}{l}23.53 \\
(4.95)\end{array}$ & $\begin{array}{l}29.10 \\
(5.49)\end{array}$ & $\begin{array}{l}31.57 \\
(5.71)\end{array}$ & $\begin{array}{l}15.70 \\
(4.09)\end{array}$ & $\begin{array}{c}11.7 \\
(3.56)\end{array}$ & $\begin{array}{c}7.1 \\
(2.85)\end{array}$ & $\begin{array}{c}4.4 \\
(2.32)\end{array}$ & $\begin{array}{l}31.57 \\
(5.71)\end{array}$ & $\begin{array}{c}15.8 \\
(4.10)\end{array}$ \\
\hline & 2 & Indrayani & $\begin{array}{c}4.9 \\
(2.43)\end{array}$ & $\begin{array}{c}7.89 \\
(2.98)\end{array}$ & $\begin{array}{l}12.46 \\
(3.67)\end{array}$ & $\begin{array}{c}14.73 \\
(3.9)\end{array}$ & $\begin{array}{l}21.07 \\
(4.70)\end{array}$ & $\begin{array}{l}23.90 \\
(4.99)\end{array}$ & $\begin{array}{c}32.8 \\
(5.81)\end{array}$ & $\begin{array}{l}36.70 \\
(6.01)\end{array}$ & $\begin{array}{l}22.30 \\
(5.23)\end{array}$ & $\begin{array}{l}12.79 \\
(3.71)\end{array}$ & $\begin{array}{c}7.7 \\
(2.97)\end{array}$ & $\begin{array}{c}4.9 \\
(2.43)\end{array}$ & $\begin{array}{l}36.70 \\
(6.01)\end{array}$ & $\begin{array}{l}16.44 \\
(4.18)\end{array}$ \\
\hline & 3 & Bhogavati & $\begin{array}{c}5.2 \\
(2.49)\end{array}$ & $\begin{array}{c}7.75 \\
(2.96)\end{array}$ & $\begin{array}{l}13.18 \\
(3.77) \\
\end{array}$ & $\begin{array}{c}15.44 \\
(4.0)\end{array}$ & $\begin{array}{l}22.30 \\
(4.83)\end{array}$ & $\begin{array}{l}26.33 \\
(5.23)\end{array}$ & $\begin{array}{c}36.0 \\
(6.03)\end{array}$ & $\begin{array}{l}38.40 \\
(6.24)\end{array}$ & $\begin{array}{l}21.86 \\
(4.78)\end{array}$ & $\begin{array}{l}14.70 \\
(3.96)\end{array}$ & $\begin{array}{c}9.2 \\
(3.19)\end{array}$ & $\begin{array}{c}5.2 \\
(2.49)\end{array}$ & $\begin{array}{l}38.40 \\
(6.24)\end{array}$ & $\begin{array}{c}17.4 \\
(4.31)\end{array}$ \\
\hline & 4 & Phule Radha & $\begin{array}{c}6.1 \\
(2.67)\end{array}$ & $\begin{array}{c}9.2 \\
(3.19)\end{array}$ & $\begin{array}{l}13.93 \\
(3.86\end{array}$ & $\begin{array}{l}17.27 \\
(4.2)\end{array}$ & $\begin{array}{l}24.18 \\
(5.02)\end{array}$ & $\begin{array}{l}29.1( \\
5.49)\end{array}$ & $\begin{array}{l}37.13 \\
(6.17) \\
\end{array}$ & $\begin{array}{l}39.93 \\
(6.37) \\
\end{array}$ & $\begin{array}{l}24.07 \\
.(5.0)\end{array}$ & $\begin{array}{l}15.70 \\
(3.56)\end{array}$ & $\begin{array}{c}7.1 \\
(2.85)\end{array}$ & $\begin{array}{c}6.1 \\
(2.67)\end{array}$ & $\begin{array}{l}39.93 \\
(6.37)\end{array}$ & $\begin{array}{l}19.46 \\
(4.35)\end{array}$ \\
\hline \multirow{7}{*}{$\begin{array}{c}S_{2}-28^{\text {th }} \\
M W\end{array}$} & Sr. & Variety & \multicolumn{11}{|c|}{ Mean of observations taken on SMW } & \multirow{2}{*}{\multicolumn{2}{|c|}{ Range }} & Mean \\
\hline & No & & 30 & 31 & 32 & 33 & 34 & 35 & 36 & 37 & 38 & 39 & 40 & & & \\
\hline & \multicolumn{13}{|c|}{ No. of insects /40 hills } & Min & Max & \\
\hline & 1 & $\begin{array}{c}\text { Phule } \\
\text { Samrudhi }\end{array}$ & $\begin{array}{c}3.9 \\
(2.2)\end{array}$ & $\begin{array}{c}4.4 \\
(2.32)\end{array}$ & $\begin{array}{c}11.7 \\
(3.56)\end{array}$ & $\begin{array}{l}13.93 \\
(3.85)\end{array}$ & $\begin{array}{r}14.70 \\
(3.96)\end{array}$ & $\begin{array}{l}23.53 \\
(14.5)\end{array}$ & $\begin{array}{l}20.90 \\
(4.68)\end{array}$ & $\begin{array}{c}11.7 \\
(3.56)\end{array}$ & $\begin{array}{c}9.3 \\
(3.21)\end{array}$ & $\begin{array}{c}4.4 \\
(2.32) \\
\end{array}$ & $\begin{array}{c}3.9 \\
(2.21)\end{array}$ & $\begin{array}{c}3.9 \\
(2.2)\end{array}$ & $\begin{array}{l}23.53 \\
(4.5)\end{array}$ & $\begin{array}{l}11.12 \\
(3.32)\end{array}$ \\
\hline & 2 & Indrayani & $\begin{array}{c}5.2 \\
(2.48)\end{array}$ & $\begin{array}{c}6.8 \\
(2.79)\end{array}$ & $\begin{array}{l}12.79 \\
(3.71)\end{array}$ & $\begin{array}{c}14.73 \\
(3.9)\end{array}$ & $\begin{array}{l}15.70 \\
(4.09)\end{array}$ & $\begin{array}{l}23.90 \\
(4.99)\end{array}$ & $\begin{array}{l}21.07 \\
(4.76)\end{array}$ & $\begin{array}{c}15.7 \\
(4.09)\end{array}$ & $\begin{array}{l}10.13 \\
(3.34)\end{array}$ & $\begin{array}{c}6.8 \\
(2.79)\end{array}$ & $\begin{array}{c}4.4 \\
(2.32)\end{array}$ & $\begin{array}{c}4.4 \\
(2.32)\end{array}$ & $\begin{array}{l}23.90 \\
(4.99)\end{array}$ & $\begin{array}{l}12.74 \\
(3.66)\end{array}$ \\
\hline & 3 & Bhogavati & $\begin{array}{c}4.9 \\
(2.43) \\
\end{array}$ & $\begin{array}{c}8.9 \\
(3.05)\end{array}$ & $\begin{array}{c}14.7 \\
(3.93) \\
\end{array}$ & $\begin{array}{l}15.44 \\
(4.05) \\
\end{array}$ & $\begin{array}{l}20.90 \\
(4.68)\end{array}$ & $\begin{array}{l}26.33 \\
(5.23) \\
\end{array}$ & $\begin{array}{l}22.30 \\
(4.83) \\
\end{array}$ & $\begin{array}{c}17.9 \\
(4.35) \\
\end{array}$ & $\begin{array}{l}10.28 \\
(3.36) \\
\end{array}$ & $\begin{array}{c}5.9 \\
(3.05) \\
\end{array}$ & $\begin{array}{c}4.9 \\
(2.43)\end{array}$ & $\begin{array}{c}4.9 \\
(2.43)\end{array}$ & $\begin{array}{l}26.33 \\
(5.23)\end{array}$ & $\begin{array}{l}13.85 \\
(3.78)\end{array}$ \\
\hline & 4 & Phule Radha & $\begin{array}{c}6.7 \\
(2.77)\end{array}$ & $\begin{array}{c}9.33 \\
(3.21)\end{array}$ & $\begin{array}{l}15.76 \\
(4.09)\end{array}$ & $\begin{array}{l}17.27 \\
(4.27)\end{array}$ & $\begin{array}{l}21.07 \\
(4.76)\end{array}$ & $\begin{array}{l}29.07 \\
(5.49)\end{array}$ & $\begin{array}{l}24.18 \\
(5.02)\end{array}$ & $\begin{array}{l}21.07 \\
(4.70)\end{array}$ & $\begin{array}{l}12.79 \\
(3.71)\end{array}$ & $\begin{array}{c}9.33 \\
(3.21)\end{array}$ & $\begin{array}{c}6.8 \\
(2.79)\end{array}$ & $\begin{array}{c}6.7 \\
(2.77)\end{array}$ & $\begin{array}{l}29.07 \\
(5.49)\end{array}$ & $\begin{array}{l}15.76 \\
(4.26)\end{array}$ \\
\hline \multirow{7}{*}{$\begin{array}{c}\mathrm{S}_{3}-30^{\text {th }} \\
\mathrm{MW}\end{array}$} & Sr. & Variety & \multicolumn{11}{|c|}{ Mean of observations taken on SMW } & \multirow{2}{*}{\multicolumn{2}{|c|}{ Range }} & Mean \\
\hline & No & & 32 & 33 & 34 & 35 & 36 & 37 & 38 & 39 & 40 & 41 & 42 & & & \\
\hline & \multicolumn{13}{|c|}{ No. of insects /40 hills } & Min & Max & \\
\hline & 1 & $\begin{array}{c}\text { Phule } \\
\text { Samrudhi }\end{array}$ & $\begin{array}{c}3.8 \\
(2.1) \\
\end{array}$ & $\begin{array}{c}9.3 \\
(3.21)\end{array}$ & $\begin{array}{c}7.1 \\
(2.85) \\
\end{array}$ & $\begin{array}{l}13.18 \\
(3.77) \\
\end{array}$ & $\begin{array}{c}11.7 \\
(3.56)\end{array}$ & $\begin{array}{c}37.80( \\
6.31)\end{array}$ & $\begin{array}{c}29.1 \\
(5.49) \\
\end{array}$ & $\begin{array}{l}24.07 \\
(5.01) \\
\end{array}$ & $\begin{array}{l}21.30 \\
(4.72) \\
\end{array}$ & $\begin{array}{l}19.90 \\
(4.57) \\
\end{array}$ & $\begin{array}{c}5.1 \\
(2.46) \\
\end{array}$ & $\begin{array}{c}3.8 \\
(2.2) \\
\end{array}$ & $\begin{array}{r}37.80 \\
(6.31)\end{array}$ & $\begin{array}{l}14.47 \\
(3.82)\end{array}$ \\
\hline & 2 & Indrayani & $\begin{array}{c}4.1 \\
(2.25)\end{array}$ & $\begin{array}{c}9.13 \\
(3.18)\end{array}$ & $\begin{array}{c}7.7 \\
(2.9)\end{array}$ & $\begin{array}{l}14.70 \\
(3.96)\end{array}$ & $\begin{array}{l}12.79 \\
(3.71)\end{array}$ & $\begin{array}{l}38.53 \\
(6.37)\end{array}$ & $\begin{array}{l}31.57 \\
(5.71)\end{array}$ & $\begin{array}{l}24.07 \\
(5.01)\end{array}$ & $\begin{array}{l}21.90 \\
(4.79)\end{array}$ & $\begin{array}{l}21.90 \\
(4.79)\end{array}$ & $\begin{array}{c}6.4 \\
(2.72)\end{array}$ & $\begin{array}{c}4.1 \\
(2.25)\end{array}$ & $\begin{array}{l}38.52 \\
(6.37)\end{array}$ & $\begin{array}{c}17.7 \\
(4.41)\end{array}$ \\
\hline & 3 & Bhogavati & $\begin{array}{c}5.1 \\
(2.4)\end{array}$ & $\begin{array}{c}10.28 \\
(3.36)\end{array}$ & $\begin{array}{c}9.2 \\
(3.19)\end{array}$ & $\begin{array}{l}17.27 \\
(4.27)\end{array}$ & $\begin{array}{l}13.70 \\
(3.96)\end{array}$ & $\begin{array}{l}39.90 \\
(6.63)\end{array}$ & $\begin{array}{c}36.1 \\
(6.09)\end{array}$ & $\begin{array}{l}26.33 \\
(5.23)\end{array}$ & $\begin{array}{l}22.30 \\
(4.83)\end{array}$ & $\begin{array}{l}23.26 \\
(4.93)\end{array}$ & $\begin{array}{c}7.5 \\
(2.9)\end{array}$ & $\begin{array}{c}5.1 \\
(2.4)\end{array}$ & $\begin{array}{l}42.90 \\
(6.63)\end{array}$ & $\begin{array}{l}19.52 \\
(4.51)\end{array}$ \\
\hline & 4 & Phule Radha & $\begin{array}{c}6.7 \\
(2.79)\end{array}$ & $\begin{array}{l}12.79 \\
(3.71)\end{array}$ & $\begin{array}{c}9.4 \\
(3.24)\end{array}$ & $\begin{array}{l}19.90 \\
(4.57)\end{array}$ & $\begin{array}{l}14.70 \\
(4.09)\end{array}$ & $\begin{array}{l}40.30 \\
(6.80)\end{array}$ & $\begin{array}{c}39.6 \\
(6.37)\end{array}$ & $\begin{array}{c}29.4 \\
(5.51)\end{array}$ & $\begin{array}{l}24.18 \\
(5.02)\end{array}$ & $\begin{array}{c}24.7 \\
(5.01)\end{array}$ & $\begin{array}{c}8.9 \\
(3.15)\end{array}$ & $\begin{array}{c}6.7 \\
(2.79)\end{array}$ & $\begin{array}{l}40.30 \\
(6.80)\end{array}$ & $\begin{array}{l}21.52 \\
(4.88)\end{array}$ \\
\hline
\end{tabular}


Int.J.Curr.Microbiol.App.Sci (2021) 10(10): 108-117

Table.2 Contd....

\begin{tabular}{|c|c|c|c|c|c|c|c|c|c|c|c|c|c|c|c|c|}
\hline \multirow{7}{*}{$\begin{array}{c}\mathrm{S}_{4}-32^{\text {th }} \\
\mathrm{MW}\end{array}$} & \multirow{2}{*}{$\begin{array}{l}\text { Sr. } \\
\text { No }\end{array}$} & \multirow[t]{2}{*}{ Variety } & \multicolumn{11}{|c|}{ Mean of observations taken on SMW } & \multirow{2}{*}{\multicolumn{2}{|c|}{ Range }} & \multirow[t]{3}{*}{ Mean } \\
\hline & & & 34 & 35 & 36 & 37 & 38 & 39 & 40 & 41 & 42 & 43 & 44 & & & \\
\hline & \multicolumn{13}{|c|}{ No. of insects /40 hills } & Min & Max & \\
\hline & 1 & Phule Samrudhi & $\begin{array}{c}4.5 \\
(2.32)\end{array}$ & $\begin{array}{c}9.2 \\
(3.1)\end{array}$ & $\begin{array}{l}22.30 \\
(4.83)\end{array}$ & $\begin{array}{c}36 \\
(6.0)\end{array}$ & $\begin{array}{l}29.10 \\
(5.49)\end{array}$ & $\begin{array}{c}25 \\
(5.12)\end{array}$ & $\begin{array}{c}23.8 \\
(4.98)\end{array}$ & $\begin{array}{l}15.70 \\
(4.09)\end{array}$ & $\begin{array}{c}7.1 \\
(2.85)\end{array}$ & $\begin{array}{c}3.9 \\
(2.21)\end{array}$ & $\begin{array}{c}3.1 \\
(2.04)\end{array}$ & $\begin{array}{c}3.1 \\
(2.04)\end{array}$ & $\begin{array}{c}36 \\
(6.0)\end{array}$ & $\begin{array}{l}16.32 \\
(4.12)\end{array}$ \\
\hline & 2 & Indrayani & $\begin{array}{c}6.7 \\
(2.79)\end{array}$ & $\begin{array}{l}15.70 \\
(4.09)\end{array}$ & $\begin{array}{c}21.80 \\
(4.7)\end{array}$ & $\begin{array}{c}38.80 \\
(6.3)\end{array}$ & $\begin{array}{l}35.90 \\
(6.07) \\
\end{array}$ & $\begin{array}{l}29.40 \\
(5.51)\end{array}$ & $\begin{array}{l}23.66 \\
(4.97) \\
\end{array}$ & $\begin{array}{l}17.93 \\
(4.35) \\
\end{array}$ & $\begin{array}{c}7.7 \\
(2.97) \\
\end{array}$ & $\begin{array}{c}4.9 \\
(2.43) \\
\end{array}$ & $\begin{array}{c}4.1 \\
(2.34)\end{array}$ & $\begin{array}{c}4.1 \\
(2.34)\end{array}$ & $\begin{array}{c}38.80 \\
(6.3)\end{array}$ & $\begin{array}{l}18.79 \\
(4.65)\end{array}$ \\
\hline & 3 & Bhogavati & $\begin{array}{c}8.9 \\
(3.05)\end{array}$ & $\begin{array}{c}17.9 \\
(4.35)\end{array}$ & $\begin{array}{c}32.9 \\
(5.81)\end{array}$ & $\begin{array}{l}43.4 \\
(6.6)\end{array}$ & $\begin{array}{l}37.13 \\
(6.17)\end{array}$ & $\begin{array}{l}34.50 \\
(5.96)\end{array}$ & $\begin{array}{l}23.93 \\
(4.93)\end{array}$ & $\begin{array}{l}21.86 \\
(4.78)\end{array}$ & $\begin{array}{c}6.5 \\
(2.75)\end{array}$ & $\begin{array}{c}4.4 \\
(2.32)\end{array}$ & $\begin{array}{c}3.8 \\
(2.12)\end{array}$ & $\begin{array}{c}3.8 \\
(2.12)\end{array}$ & $\begin{array}{l}43.4 \\
(6.6)\end{array}$ & $\begin{array}{l}21.06 \\
(4.54)\end{array}$ \\
\hline & 4 & Phule Radha & $\begin{array}{c}9.33 \\
(3.21)\end{array}$ & $\begin{array}{l}21.07 \\
(4.70)\end{array}$ & $\begin{array}{l}38.20 \\
(6.26)\end{array}$ & $\begin{array}{c}56.50 \\
(7.5)\end{array}$ & $\begin{array}{l}45.30 \\
(6.80)\end{array}$ & $\begin{array}{l}42.90 \\
(6.63)\end{array}$ & $\begin{array}{l}39.53 \\
(6.37)\end{array}$ & $\begin{array}{l}24.07 \\
(5.01)\end{array}$ & $\begin{array}{c}7.89 \\
(2.98)\end{array}$ & $\begin{array}{c}5.80 \\
(2.61)\end{array}$ & $\begin{array}{c}4.3 \\
(2.28)\end{array}$ & $\begin{array}{c}4.3 \\
(2.28)\end{array}$ & $\begin{array}{c}56.50 \\
(7.5)\end{array}$ & $\begin{array}{l}26.80 \\
(5.48)\end{array}$ \\
\hline
\end{tabular}


Table.3 Seasonal incidence of brown plant hopper association with rice (2017)

\begin{tabular}{|c|c|c|c|c|c|c|c|c|c|c|c|c|c|c|c|c|}
\hline \multirow{7}{*}{$\begin{array}{c}S_{1}-26^{\text {th }} \\
M W\end{array}$} & Sr. & Variety & \multicolumn{11}{|c|}{ Mean of observations taken on SMW } & \multirow{2}{*}{\multicolumn{2}{|c|}{ Range }} & \multirow[t]{3}{*}{ Mean } \\
\hline & No & & 28 & 29 & 30 & 31 & 32 & 33 & 34 & 35 & 36 & 37 & 38 & & & \\
\hline & \multicolumn{13}{|c|}{ No. of insects $/ 40$ hills } & Min & Max & \\
\hline & 1 & Phule Samrudhi & $\begin{array}{c}4.1 \\
(2.21)\end{array}$ & $\begin{array}{c}6.0 \\
(2.67)\end{array}$ & $\begin{array}{c}8.4 \\
(3.15)\end{array}$ & $\begin{array}{c}9.7 \\
(3.41)\end{array}$ & $\begin{array}{c}12.1 \\
(3.32)\end{array}$ & $\begin{array}{l}13.93 \\
(3.85)\end{array}$ & $\begin{array}{l}20.90 \\
(2.68)\end{array}$ & $\begin{array}{c}39.4 \\
(5.67)\end{array}$ & $\begin{array}{l}31.57 \\
(5.7)\end{array}$ & $\begin{array}{l}22.13 \\
(4.95)\end{array}$ & $\begin{array}{l}21.30 \\
(4.72)\end{array}$ & $\begin{array}{c}4.1 \\
(2.21)\end{array}$ & $\begin{array}{c}39.4 \\
(5.67)\end{array}$ & $\begin{array}{l}17.42 \\
(4.42)\end{array}$ \\
\hline & 2 & Indrayani & $\begin{array}{c}6.9 \\
(2.81)\end{array}$ & $\begin{array}{c}7.5 \\
(2.92)\end{array}$ & $\begin{array}{c}9.9 \\
(3.25)\end{array}$ & $\begin{array}{l}10.28 \\
(3.36)\end{array}$ & $\begin{array}{c}13.6 \\
(3.72)\end{array}$ & $\begin{array}{l}15.44 \\
(4.05)\end{array}$ & $\begin{array}{l}24.18 \\
(5.02)\end{array}$ & $\begin{array}{l}45.30 \\
(6.80)\end{array}$ & $\begin{array}{l}38.40 \\
(6.28)\end{array}$ & $\begin{array}{c}27.4 \\
(5.41)\end{array}$ & $\begin{array}{l}24.07 \\
(5.01)\end{array}$ & $\begin{array}{c}6.9 \\
(2.81)\end{array}$ & $\begin{array}{l}45.30 \\
(6.80)\end{array}$ & $\begin{array}{l}18.28 \\
(4.28)\end{array}$ \\
\hline & 3 & Bhogavati & $\begin{array}{c}4.9 \\
(12.43 \\
)\end{array}$ & $\begin{array}{c}7.2 \\
(2.81)\end{array}$ & $\begin{array}{c}9.2 \\
(3.19)\end{array}$ & $\begin{array}{l}10.54 \\
(3.38\end{array}$ & $\begin{array}{l}14.73 \\
(3.97)\end{array}$ & $\begin{array}{l}17.27 \\
(4.27)\end{array}$ & $\begin{array}{l}22.30 \\
(4.83)\end{array}$ & $\begin{array}{c}40.2 \\
(5.78)\end{array}$ & $\begin{array}{l}32.70 \\
(5.81)\end{array}$ & $\begin{array}{l}24.18 \\
(5.02)\end{array}$ & $\begin{array}{l}22.30 \\
(4.83)\end{array}$ & $\begin{array}{c}4.9 \\
(2.43)\end{array}$ & $\begin{array}{c}40.2 \\
(5.78)\end{array}$ & $\begin{array}{l}18.68 \\
(4.48)\end{array}$ \\
\hline & 4 & Phule Radha & $\begin{array}{c}7.5 \\
(2.92)\end{array}$ & $\begin{array}{c}9.2 \\
(3.9)\end{array}$ & $\begin{array}{c}11.7 \\
(3.56 \\
)\end{array}$ & $\begin{array}{l}12.79 \\
(3.71)\end{array}$ & $\begin{array}{l}17.27 \\
(4.27)\end{array}$ & $\begin{array}{l}21.07 \\
(4.70)\end{array}$ & $\begin{array}{l}26.33 \\
(5.23)\end{array}$ & $\begin{array}{l}46.30 \\
(7.21)\end{array}$ & $\begin{array}{l}39.53 \\
(6.37)\end{array}$ & $\begin{array}{c}29.1 \\
(5.49)\end{array}$ & $\begin{array}{l}26.33 \\
(5.29)\end{array}$ & $\begin{array}{c}7.5 \\
(2.92)\end{array}$ & $\begin{array}{l}46.30 \\
(7.21)\end{array}$ & $\begin{array}{l}20.47 \\
(2.46)\end{array}$ \\
\hline \multirow{7}{*}{$\begin{array}{c}\mathrm{S}_{2}-28^{\text {th }} \\
\mathrm{MW}\end{array}$} & Sr. & Variety & \multicolumn{11}{|c|}{ Mean of observations taken on SMW } & \multirow{2}{*}{\multicolumn{2}{|c|}{ Range }} & Mean \\
\hline & No & & 30 & 31 & 32 & 33 & 34 & 35 & 36 & 37 & 38 & 39 & 40 & & & \\
\hline & \multicolumn{13}{|c|}{ No. of insects /40 hills } & Min & Max & \\
\hline & 1 & $\begin{array}{c}\text { Phule } \\
\text { Samrudhi }\end{array}$ & $\begin{array}{c}4.0 \\
(2.21)\end{array}$ & $\begin{array}{c}7.1 \\
(2 . .8)\end{array}$ & $\begin{array}{c}9.3 \\
(3.21)\end{array}$ & $\begin{array}{c}11.7 \\
(3.56)\end{array}$ & $\begin{array}{l}13.18 \\
(3.77)\end{array}$ & $\begin{array}{l}20.90 \\
(4.68)\end{array}$ & $\begin{array}{l}13.93 \\
(3.86)\end{array}$ & $\begin{array}{l}10.28 \\
(3.36)\end{array}$ & $\begin{array}{c}5.80 \\
(2.61)\end{array}$ & $\begin{array}{c}4.4 \\
(2.32)\end{array}$ & $\begin{array}{c}3.3 \\
(2.18)\end{array}$ & $\begin{array}{c}3.2 \\
(2.18)\end{array}$ & $\begin{array}{l}20.90 \\
(4.68)\end{array}$ & $\begin{array}{c}9.49 \\
(3.22)\end{array}$ \\
\hline & 2 & Indrayani & $\begin{array}{c}5.2 \\
(2.49)\end{array}$ & $\begin{array}{c}9.2 \\
(3.19)\end{array}$ & $\begin{array}{l}10.28 \\
(3.36)\end{array}$ & $\begin{array}{l}14.70 \\
(3.96)\end{array}$ & $\begin{array}{l}17.27 \\
(4.27)\end{array}$ & $\begin{array}{l}22.30 \\
(4.96)\end{array}$ & $\begin{array}{l}15.44 \\
(4.05)\end{array}$ & $\begin{array}{c}13.18 \\
(3.7)\end{array}$ & $\begin{array}{c}7.75 \\
(2.96)\end{array}$ & $\begin{array}{c}5.2 \\
(2.4)\end{array}$ & $\begin{array}{c}4.8 \\
(2.41)\end{array}$ & $\begin{array}{c}4.8 \\
(2.41\end{array}$ & $\begin{array}{l}22.30 \\
(4.96)\end{array}$ & $\begin{array}{c}9.7 \\
(3.41)\end{array}$ \\
\hline & 3 & Bhogavati & $\begin{array}{c}4.9 \\
(2.43)\end{array}$ & $\begin{array}{c}7.7 \\
(2.97)\end{array}$ & $\begin{array}{l}10.13 \\
(3.34)\end{array}$ & $\begin{array}{l}12.79 \\
(3.7)\end{array}$ & $\begin{array}{l}14.70 \\
(3.96)\end{array}$ & $\begin{array}{l}21.07 \\
(4.70)\end{array}$ & $\begin{array}{l}14.73 \\
(3.97)\end{array}$ & $\begin{array}{l}12.46 \\
(3.67)\end{array}$ & $\begin{array}{c}7.89 \\
(2.98)\end{array}$ & $\begin{array}{c}4.9 \\
(2.43)\end{array}$ & $\begin{array}{c}4.4 \\
(2.32)\end{array}$ & $\begin{array}{c}4.4 \\
(2.32)\end{array}$ & $\begin{array}{l}21.07 \\
(4.70)\end{array}$ & $\begin{array}{l}10.51 \\
(3.54)\end{array}$ \\
\hline & 4 & Phule Radha & $\begin{array}{c}5.6 \\
(2.61)\end{array}$ & $\begin{array}{c}9.5 \\
(3.24)\end{array}$ & $\begin{array}{l}12.79 \\
(3.71)\end{array}$ & $\begin{array}{r}15.70 \\
(4.09)\end{array}$ & $\begin{array}{l}19.90 \\
(4.57)\end{array}$ & $\begin{array}{l}24.18 \\
(5.02)\end{array}$ & $\begin{array}{l}17.27 \\
(4.27)\end{array}$ & $\begin{array}{l}13.93 \\
(3.86)\end{array}$ & $\begin{array}{c}9.2 \\
(3.19)\end{array}$ & $\begin{array}{c}6.1 \\
(2.67)\end{array}$ & $\begin{array}{c}5.4 \\
(2.49)\end{array}$ & $\begin{array}{c}5.4 \\
(2.49)\end{array}$ & $\begin{array}{l}24.18 \\
(5.02)\end{array}$ & $\begin{array}{c}12.68 \\
(3.68)\end{array}$ \\
\hline \multirow{7}{*}{$\begin{array}{c}\mathrm{S}_{3}-\mathbf{3 0}^{\text {th }} \\
\mathrm{MW}\end{array}$} & Sr. & Variety & \multicolumn{11}{|c|}{ Mean of observations taken on SMW } & \multirow{2}{*}{\multicolumn{2}{|c|}{ Range }} & Mean \\
\hline & No & & 32 & 33 & 34 & 35 & 36 & 37 & 38 & 39 & 40 & 41 & 42 & & & \\
\hline & \multicolumn{13}{|c|}{ No. of insects $/ 40$ hills } & Min & Max & \\
\hline & 1 & Phule Samrudhi & $\begin{array}{c}4.9 \\
(2.43)\end{array}$ & $\begin{array}{c}6.1 \\
(2.67)\end{array}$ & $\begin{array}{c}11.7 \\
(3.56)\end{array}$ & $\begin{array}{l}14.70 \\
(3.9)\end{array}$ & $\begin{array}{c}20.90 \\
(4.6)\end{array}$ & $\begin{array}{c}36 \\
(6.08)\end{array}$ & $\begin{array}{c}29.1 \\
(5.49)\end{array}$ & $\begin{array}{c}28.4 \\
(5.37)\end{array}$ & $\begin{array}{l}29.10 \\
(5.49)\end{array}$ & $\begin{array}{c}17.7 \\
(3.56)\end{array}$ & $\begin{array}{c}4.4 \\
(2.32)\end{array}$ & $\begin{array}{c}4.4 \\
(2.32)\end{array}$ & $\begin{array}{c}36 \\
(6.08)\end{array}$ & $\begin{array}{l}18.45 \\
(4.48)\end{array}$ \\
\hline & 2 & Indrayani & $\begin{array}{c}5.2 \\
(2.49)\end{array}$ & $\begin{array}{c}7.1 \\
(2.85)\end{array}$ & $\begin{array}{c}9.5 \\
(3.24)\end{array}$ & $\begin{array}{l}17.27 \\
(4.2)\end{array}$ & $\begin{array}{l}22.30 \\
(4.83)\end{array}$ & $\begin{array}{l}38.80 \\
(6.31)\end{array}$ & $\begin{array}{l}31.57 \\
(15.7)\end{array}$ & $\begin{array}{c}30.2 \\
(5.56)\end{array}$ & $\begin{array}{c}29.1 \\
(5.49)\end{array}$ & $\begin{array}{c}15.7 \\
(4.09)\end{array}$ & $\begin{array}{c}6.8 \\
(2.79)\end{array}$ & $\begin{array}{c}5.2 \\
(2.49)\end{array}$ & $\begin{array}{l}38.80 \\
(6.31)\end{array}$ & $\begin{array}{l}19.41 \\
(4.3)\end{array}$ \\
\hline & 3 & Bhogavati & $\begin{array}{c}5.7 \\
(2.62)\end{array}$ & $\begin{array}{c}8.1 \\
(3.01)\end{array}$ & $\begin{array}{l}12.79 \\
(3.71)\end{array}$ & $\begin{array}{c}19.90 \\
(4.5)\end{array}$ & $\begin{array}{c}24.18 \\
(5.0)\end{array}$ & $\begin{array}{l}38.40 \\
(6.28)\end{array}$ & $\begin{array}{c}36.1 \\
(6.09)\end{array}$ & $\begin{array}{c}29.1 \\
(5.49)\end{array}$ & $\begin{array}{l}32.8 \\
(5.8)\end{array}$ & $\begin{array}{c}17.9 \\
(4.35) \\
\end{array}$ & $\begin{array}{c}8.9 \\
(3.05)\end{array}$ & $\begin{array}{c}5.7 \\
(2.62) \\
\end{array}$ & $\begin{array}{l}38.40 \\
(6.28)\end{array}$ & $\begin{array}{l}21.26 \\
(4.78)\end{array}$ \\
\hline & 4 & Phule Radha & $\begin{array}{c}6.8 \\
(2.79)\end{array}$ & $\begin{array}{c}8.9 \\
(3.15)\end{array}$ & $\begin{array}{l}15.70 \\
(4.09)\end{array}$ & $\begin{array}{l}20.90 \\
(4.6)\end{array}$ & $\begin{array}{l}26.33 \\
(15.3)\end{array}$ & $\begin{array}{c}41.2 \\
(6.62)\end{array}$ & $\begin{array}{c}39.5 \\
(6.37)\end{array}$ & $\begin{array}{l}31.57 \\
(5.71)\end{array}$ & $\begin{array}{l}37.13 \\
(6.17)\end{array}$ & $\begin{array}{l}21.07 \\
(4.70)\end{array}$ & $\begin{array}{c}9.3 \\
(3.21)\end{array}$ & $\begin{array}{c}6.8 \\
(2.79)\end{array}$ & $\begin{array}{c}41.2 \\
(6.62)\end{array}$ & $\begin{array}{r}23.49 \\
(4.96)\end{array}$ \\
\hline
\end{tabular}


Int.J.Curr.Microbiol.App.Sci (2021) 10(10): 108-117

Table.3 Contd....

\begin{tabular}{|c|c|c|c|c|c|c|c|c|c|c|c|c|c|c|c|c|}
\hline \multirow{7}{*}{$\begin{array}{c}\mathrm{S}_{4}-\mathbf{3 2}^{\text {nd }} \\
\mathrm{MW}\end{array}$} & \multirow{2}{*}{$\begin{array}{l}\text { Sr. } \\
\text { No }\end{array}$} & \multirow[t]{2}{*}{ Variety } & \multicolumn{11}{|c|}{ Mean of observations taken on SMW } & \multirow{2}{*}{\multicolumn{2}{|c|}{ Range }} & \multirow[t]{2}{*}{ Mean } \\
\hline & & & 34 & 35 & 36 & 37 & 38 & 39 & 40 & 41 & 42 & 43 & 44 & & & \\
\hline & \multicolumn{13}{|c|}{ No. of insects /40 hills } & Min & Max & \\
\hline & 1 & Phule Samrudhi & $\begin{array}{c}5.2 \\
(2.49)\end{array}$ & $\begin{array}{c}14.70 \\
(3.96)\end{array}$ & $\begin{array}{l}21.07 \\
(4.70)\end{array}$ & $\begin{array}{c}39.53 \\
(6.3)\end{array}$ & $\begin{array}{l}35.57 \\
(6.01)\end{array}$ & $\begin{array}{l}31.57 \\
(5.71)\end{array}$ & $\begin{array}{c}29.1 \\
(5.49)\end{array}$ & $\begin{array}{l}23.53 \\
(4.95)\end{array}$ & $\begin{array}{l}20.90 \\
(4.68)\end{array}$ & $\begin{array}{l}13.93 \\
(3.86)\end{array}$ & $\begin{array}{c}10.23 \\
(3.36)\end{array}$ & $\begin{array}{c}5.2 \\
(2.49)\end{array}$ & $\begin{array}{c}39.5 \\
(6.37)\end{array}$ & $\begin{array}{l}22.30 \\
(4.96)\end{array}$ \\
\hline & 2 & Indrayani & $\begin{array}{c}8.9 \\
(3.05) \\
\end{array}$ & $\begin{array}{l}15.70 \\
(4.09) \\
\end{array}$ & $\begin{array}{l}23.90 \\
(4.99) \\
\end{array}$ & $\begin{array}{l}40.1 \\
(6.4)\end{array}$ & $\begin{array}{c}32.70 \\
(5.8)\end{array}$ & $\begin{array}{c}36.0 \\
(6.08) \\
\end{array}$ & $\begin{array}{c}29.4 \\
(5.53) \\
\end{array}$ & $\begin{array}{l}23.90 \\
(4.99) \\
\end{array}$ & $\begin{array}{l}21.07 \\
(4.70) \\
\end{array}$ & $\begin{array}{l}14.73 \\
(3.97) \\
\end{array}$ & $\begin{array}{l}12.46 \\
(3.67) \\
\end{array}$ & $\begin{array}{c}8.9 \\
(3.05) \\
\end{array}$ & $\begin{array}{c}40.1 \\
(6.43) \\
\end{array}$ & $\begin{array}{l}23.53 \\
(4.95)\end{array}$ \\
\hline & 3 & Bhogavati & $\begin{array}{c}9.2 \\
(3.19) \\
\end{array}$ & $\begin{array}{l}10.90 \\
(4.68) \\
\end{array}$ & $\begin{array}{c}22.7 \\
(4.78) \\
\end{array}$ & $\begin{array}{c}42.90 \\
(6.6)\end{array}$ & $\begin{array}{c}38.40 \\
(6.2)\end{array}$ & $\begin{array}{l}36.80 \\
(6.12) \\
\end{array}$ & $\begin{array}{c}32.1 \\
(5.82) \\
\end{array}$ & $\begin{array}{l}26.38 \\
(5.23) \\
\end{array}$ & $\begin{array}{l}22.30 \\
(4.83) \\
\end{array}$ & $\begin{array}{l}15.44 \\
(4.05) \\
\end{array}$ & $\begin{array}{c}13.7 \\
(3.77) \\
\end{array}$ & $\begin{array}{c}9.2 \\
(3.19) \\
\end{array}$ & $\begin{array}{l}42.90 \\
(6.63) \\
\end{array}$ & $\begin{array}{l}24.62 \\
(5.36) \\
\end{array}$ \\
\hline & 4 & Phule Radha & $\begin{array}{l}10.23 \\
(3.36)\end{array}$ & $\begin{array}{l}21.27 \\
(4.70)\end{array}$ & $\begin{array}{c}29.1 \\
(5.49)\end{array}$ & $\begin{array}{l}56.2 \\
(7.9)\end{array}$ & $\begin{array}{c}39.53 \\
(6.3)\end{array}$ & $\begin{array}{c}48.2 \\
(7.01)\end{array}$ & $\begin{array}{l}38.40 \\
(6.28)\end{array}$ & $\begin{array}{c}31.5 \\
(5.71)\end{array}$ & $\begin{array}{l}24.18 \\
(5.02)\end{array}$ & $\begin{array}{l}17.22 \\
(4.27)\end{array}$ & $\begin{array}{l}13.93 \\
(3.86)\end{array}$ & $\begin{array}{l}10.23 \\
(3.36)\end{array}$ & $\begin{array}{c}56.2 \\
(7.96)\end{array}$ & $\begin{array}{l}30.34 \\
(5.83)\end{array}$ \\
\hline
\end{tabular}


The correlation of meteorological parameters with incidence of rice brown plant hopper was studied in 2017. The correlation coefficient (r) of brown plant hopper on rice with the weather parameters are presented in((Table.1) Average number of brown plant hopper showed significant positive correlation with maximum temperature $\left(\mathrm{r}=0.874 *, 0.627^{*}\right.$, $\left.0.724^{*}, 0.678^{*}\right)$ morning and evening relative humidity's and bright sunshine hrs $(\mathrm{r}=0.224$, $0.183,0.400,0.482$ ) during the year of 2017 , Whereas brown plant hopper population showed negative correlation with rainfall and minimum temperature during $26 \mathrm{MW}, 28$ MW, $30 \mathrm{MW}$ and $32 \mathrm{MW}$ transplanting times $(\mathrm{r}=-0.192,-0.307,-0.668$ and -0.546$),(\mathrm{r}=-$ $0.637,-0.543,-0.045$ and -0.593$)$, respectively during the year of 2017 .

It was observed that, the infestation of brown plant hopper started increasing September onwards and it was peak during $37^{\text {th }} \mathrm{MW}$. This was in agreement with the present finding. Similar results were reported by Yadav and Chander (2010) and Sujithra and Chander (2012).

Average number of brown plant hoppers showed positive correlation with maximum temperature, morning and evening relative humidity's and bright sunshine hrs Whereas, non-significant negative correlation with rainfall and minimum temperature respectively.

It was observed that, the infestation of brown plant hoppers started increasing from midaugust onwards and it was peak during $35^{\text {th }}$ to $37^{\text {th }}$ MW in RDN-99-1 $\left(\mathrm{V}_{4}\right)$. Among the rice varieties, for the year of 2016 and 2017 higher incidence was recorded RDN-99-1 and minimum was recorded on VDN-99-29 i.e Phule samruddhi. So Farmers are advised to use Phule Samrudhi variety for rice cultivation for highest production with minimum attack of pest and use $28^{\text {th }} \mathrm{MW}$ (16 July-15July) for rice transplantation.

\section{References}

Chaudhary, D. T. and Raghuraman, V. 2014. Rice yield losses caused by brown plant hopper damage to the flag leaf. Int. Rice Res. Newsl. 9(4) : 18.

Ling K C. Rice virus diseases. International Rice Research Institute, Los Banos, Philippines. 1975; 3436- 3441.

Rai, A. K. and Khan, M. A. 2002.Light trap catch of insect pest, Nephotettixvirescens (Distant) and its relation with climatic factors. Annals of Plant Protection Science. 10 (1): 17-22.

Rao N V, Maheshwari T V, Prasad P R, Naidu V G, Savithri. In Integrated Pest Managemant. Agrobios. 2003; 89.

Subhash, C., Mazhar, H., Vishwa, P., Himanshu, P., Singh, S. D., Ramesh, H. and Vinod. 2016. Effect of transplanting date and cultivars on brown plant hopper infestation in rice with climate change adaptation perspective. Proceedings of National Academy of Sciences, India. Section B Biological Sciences. 86(2):315-323.

Sujithra, T. P. and Chander, V. R. 2013. Simulation of rice brown plant hopper, Nilaparvata lugens (Stal.) population and crop-pest interactions to assess climate change impact. Article in Climatic Change. 121(2) : 15-18.

Wains, M. S., Ali, M. A., Hussain, M., Anwar, J., Zulkiffal, M. and Waseem, S. 2010. Aphid dynamics in relation to meteorological factors and various management practices in bread wheat. Journal of Plant Protection Research. 50(3):385-392.

Yadav, D. S. and Chander, Y. 2010. Agroecological zoning of brown plant hopper Nilaparvata lugens (Stål) oryza sativa $L$. incidence on rice Journal of Scientific and Industrial Research, $69: 818-822$. 


\section{How to cite this article:}

Amruta Deshmukh, S. B. Kharbade, N. D. Tamboli, V. A. Sthool and Bagade, S. V. 2021. Seasonal Abundance of Brown Plant Hopper, Nilaparvata lugens (Stal) in Rice and Correlation of Abiotic Factors Under Pune Region. Int.J.Curr.Microbiol.App.Sci. 10(10): 108-117. doi: https://doi.org/10.20546/ijcmas.2021.1010.014 